\title{
ON JETS, EXTENSIONS AND CHARACTERISTIC CLASSES II
}

\author{
HELGE MAAKESTAD
}

(Communicated by Lei Ni)

\begin{abstract}
In this paper we define the generalized Atiyah classes $c_{\mathcal{J}}(\mathcal{E})$ and $c_{\mathcal{O}_{X}}(\mathcal{E})$ of a quasi-coherent sheaf $\mathcal{E}$ with respect to a pair $(\mathcal{I}, d)$, where $\mathcal{I}$ is a left and right $\mathcal{O}_{X}$-module and $d$ a derivation. We relate this class to the structure of left and right modules on the first order jet bundle $\mathcal{J}_{\mathcal{I}}^{1}(\mathcal{E})$. In the main result of the paper we show $c_{\mathcal{O}_{X}}(\mathcal{E})=0$ if and only if there is an isomorphism $\mathcal{J}_{\mathcal{I}}^{1}(\mathcal{E})^{l e f t} \cong \mathcal{J}_{\mathcal{I}}^{1}(\mathcal{E})^{\text {right }}$ as $\mathcal{O}_{X}$-modules. We also give explicit examples where $c_{\mathcal{O}_{X}}(\mathcal{E}) \neq 0$ using jet bundles of line bundles on the projective line. Hence the classes $c_{\mathcal{J}}(\mathcal{E})$ and $c_{\mathcal{O}_{X}}(\mathcal{E})$ are nontrivial. The classes we introduce generalize the classical Atiyah class.
\end{abstract}

\section{INTRODUCTION}

The aim of this paper is to introduce and study generalized first order jet bundles and generalized Atiyah classes for quasi-coherent sheaves relative to an arbitrary morphism $\pi: X \rightarrow S$ of schemes. We define the generalized first order jet bundle $\mathcal{J}_{\mathcal{I}}^{1}(\mathcal{E})$ of $\mathcal{E}$ and the generalized Atiyah sequence

$$
0 \rightarrow \mathcal{I} \otimes_{\mathcal{O}_{X}} \mathcal{E} \rightarrow \mathcal{J}_{\mathcal{I}}^{1}(\mathcal{E}) \rightarrow \mathcal{E} \rightarrow 0 .
$$

The generalized Atiyah sequence is an exact sequence of quasi-coherent sheaves of left and right $\mathcal{O}_{X}$-modules and left and right $\mathcal{J}_{\mathcal{I}}^{1}$-modules. The sheaf $\mathcal{J}_{\mathcal{I}}^{1}$ is a sheaf of associative rings on $X$. It is an extension of $\mathcal{O}_{X}$ with a left and right quasicoherent $\mathcal{O}_{X}$-module $\mathcal{I}$ of square zero. The main result of the paper is that the sequence (1.0.1) is left split as a sequence of $\mathcal{O}_{X}$-modules if and only if the left and right structures on $\mathcal{J}_{\mathcal{I}}^{1}(\mathcal{E})$ are $\mathcal{O}_{X}$-isomorphic (see Theorem $[3.6$ ).

We give a general definition of the first order jet bundle $\mathcal{J}_{\mathcal{I}}^{1}(\mathcal{E})$ of a quasi-coherent sheaf $\mathcal{E}$ using a derivation $d$ and a left and right $\mathcal{O}_{X}$-module $\mathcal{I}$. We define generalized Atiyah classes $c_{\mathcal{J}}(\mathcal{E})$ and $c_{\mathcal{O}_{X}}(\mathcal{E})$ of $\mathcal{E}$ and relate these classes to the left and right $\mathcal{O}_{X}$ structure on $\mathcal{J}_{\mathcal{I}}^{1}(\mathcal{E})$. The generalized Atiyah class $c_{\mathcal{O}_{X}}(\mathcal{E})$ measures when there is an isomorphism

$$
\mathcal{J}_{\mathcal{I}}^{1}(\mathcal{E})^{l e f t} \cong \mathcal{J}_{\mathcal{I}}^{1}(\mathcal{E})^{r i g h t}
$$

as $\mathcal{O}_{X}$-modules (see Theorem 3.6). There is always an isomorphism

$$
\mathcal{J}_{\mathcal{I}}^{1}(\mathcal{E})^{l e f t} \cong \mathcal{J}_{\mathcal{I}}^{1}(\mathcal{E})^{\text {right }}
$$

Received by the editors January 11, 2011 and, in revised form, January 28, 2011; April 12, 2011; and June 13, 2011.

2010 Mathematics Subject Classification. Primary 14F10, 14F40.

Key words and phrases. Atiyah sequence, jet bundle, characteristic class, generalized Atiyah class, square zero extension, lifting. 
as sheaves of abelian groups. Hence the class $c_{\mathcal{O}_{X}}(\mathcal{E})$ measures when the sheaf of abelian groups $\mathcal{J}_{\mathcal{I}}^{1}(\mathcal{E})$ may be given two nonisomorphic structures as an $\mathcal{O}_{X^{-}}$ module. When $\mathcal{I}=\Omega_{X / S}^{1}$ and $d: \mathcal{O}_{X} \rightarrow \Omega_{X / S}^{1}$ is the universal derivation, it follows that the characteristic class $c_{\mathcal{O}_{X}}(\mathcal{E})$ is the classical Atiyah class as defined in 2 .

We prove in Example 3.10 that there is no isomorphism

$$
\mathcal{J}_{\mathbf{P}^{1}}^{1}(\mathcal{O}(d))^{l e f t} \neq \mathcal{J}_{\mathbf{P}^{1}}^{1}(\mathcal{O}(d))^{\text {right }}
$$

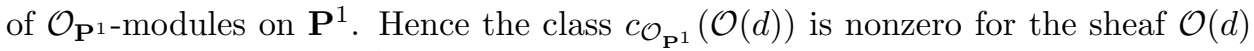
on the projective line $\mathbf{P}^{1}$ over a field $K$ of characteristic zero. If $\operatorname{char}(K)$ divides $d$, it follows that $c_{\mathcal{O}_{\mathrm{P}^{1}}}(\mathcal{O}(d))$ is zero; hence in this case there is an isomorphism

$$
\mathcal{J}_{\mathbf{P}^{1}}^{1}(\mathcal{O}(d))^{l e f t} \cong \mathcal{J}_{\mathbf{P}^{1}}^{1}(\mathcal{O}(d))^{\text {right }}
$$

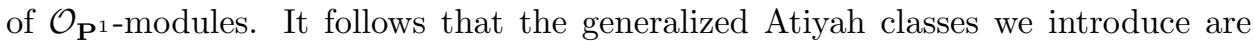
nontrivial.

In a previous paper on jet bundles (see [10]) we found many examples of jets of line bundles on the projective line where the structure as a left locally free $\mathcal{O}_{\mathbf{P}^{1}}$ module was nonisomorphic to the structure as a right locally free $\mathcal{O}_{\mathbf{P}^{1} \text {-module. In }}$ this paper we interpret this phenomenon in terms of the generalized Atiyah class.

In the first section of the paper we do all constructions in the local case. In the second part of the paper we do all constructions in the global case. In the final section of the paper we discuss Atiyah sequences and Atiyah classes relative to arbitrary morphisms of schemes and arbitrary infinitesimal extensions of $\mathcal{O}_{X}$ by a quasi-coherent left and right $\mathcal{O}_{X}$-module. We get a definition of Atiyah classes $c_{\mathcal{J}}(\mathcal{E})$ and $c_{\mathcal{O}_{X}}(\mathcal{E})$ for an arbitrary quasi-coherent sheaf $\mathcal{E}$ of $\mathcal{O}_{X}$-modules. Here $\mathcal{J}$ is an infinitesimal extension of $\mathcal{O}_{X}$ with respect to a quasi-coherent left and right $\mathcal{O}_{X}$-module $\mathcal{I}$. The construction generalizes the classical case (see Example 4.5). In the final section of the paper we construct explicit examples. We also construct (see Example 5.6) for any smooth projective scheme $X$ over the complex numbers a characteristic class $c_{\mathcal{I}}(\mathcal{E})$ in $\mathrm{K}_{0}(X)$, where $\mathrm{K}_{0}(X)$ is the Grothendieck group of locally free finite rank sheaves on $X$. There is a canonical map of Grothendieck groups

$$
\gamma: \mathrm{K}_{0}(X) \rightarrow \mathrm{KO}(X(\mathbf{R}))
$$

where $X(\mathbf{R})$ is the underlying real smooth manifold of $X$ and $\operatorname{KO}(X(\mathbf{R}))$ is the Grothendieck group of the category of real finite rank smooth vector bundles on $X(\mathbf{R})$. The class $c_{\mathcal{I}}(\mathcal{E})$ lies in the group kernel of $\gamma$ for any locally free finite rank sheaf $\mathcal{E}$ and any left and right $\mathcal{O}_{X}$-module $\mathcal{I}$.

\section{Generalized Atiyah Classes for modules over Rings}

Let in the following $\phi: A \rightarrow B$ be a unital morphism of commutative rings. Let $E$ be a $B$-module. Let $I$ be a left and right $B$-module with $a(x b)=(a x) b$ for all $a, b \in B$ and $x \in I$ and let $d \in \operatorname{Der}_{A}(B, I)$ be a derivation. This means $d(\phi(a))=0$ for all $a \in A$ and $d(a b)=a d(b)+d(a) b$ for all $a, b \in B$. In the following we will write $d(a)$ instead of $d(\phi(a))$.

Let $\mathcal{J}_{I}^{1}=I \oplus B$ with the following multiplication:

$$
(x, a)(y, b)=(x b+a y, a b) .
$$


It follows that $\mathcal{J}_{I}^{1}$ is an associative ring with multiplicative unit $\mathbf{1}=(0,1)$. Define a map $d_{I}: B \rightarrow \mathcal{J}_{I}^{1}$ by $d_{I}(b)=(d(b), 0)$. Define maps $s, t: B \rightarrow \mathcal{J}_{I}^{1}$ by

$$
t(b)=(0, b)
$$

and

$$
s(b)=(d(b), b) .
$$

Define two left actions of $B$ on $\mathcal{J}_{I}^{1}$ as follows:

$$
(x, a)_{t} b=(x, a) t(b)=(x b, a b) \text { and } b_{t}(x, a)=t(b)(x, a)=(b x, b a)
$$

and

$$
\begin{aligned}
& (x, a)_{s} b=(x, a) s(b)=(x b+a d(b), a b), \\
& b_{s}(x, a)=s(b)(x, a)=(d(b) a+b x, b a) .
\end{aligned}
$$

The ring $\mathcal{J}_{I}^{1}$ acts canonically on $I \subseteq \mathcal{J}_{I}^{1}$ as follows:

$$
(x, 0)(y, b)=(x b, 0) \text { and }(y, b)(x, 0)=(b x, 0) .
$$

In the following when we write $\operatorname{Der}_{A}^{t}\left(B, \mathcal{J}_{I}^{1}\right)$ we mean $A$-linear derivations with $B$-structure on $\mathcal{J}_{I}^{1}$ induced by $t$ from (2.0.2).

Proposition 2.1. The following hold:

(2.1.1) $\mathcal{J}_{I}^{1}$ is a square zero extension of $B$ by $I$.

(2.1.2) (2.0.2)-(2.0.4) define $\mathcal{J}_{I}^{1}$ as left and right B-modules.

(2.1.3) The map $d_{I}$ is a derivation: $d_{I} \in \operatorname{Der}_{A}^{t}\left(B, \mathcal{J}_{I}^{1}\right)$.

(2.1.4) The maps $s, t$ are ring homomorphisms and $s-t=d_{I}$.

Proof. The proof is left to the reader.

Define the map

$$
d_{\mathcal{J}}: \mathcal{J}_{I}^{1} \rightarrow I
$$

by

$$
d_{\mathcal{J}}(x, a)=x+d(a)
$$

When we write $\operatorname{Der}_{A}^{t}\left(\mathcal{J}_{I}^{1}, I\right)$ we mean derivations linear over $A$ with respect to the left action of $A$ on $\mathcal{J}_{I}^{1}$ induced by $t$ from (2.0.2).

Lemma 2.2. The following holds: $d_{\mathcal{J}} \in \operatorname{Der}_{A}^{t}\left(\mathcal{J}_{I}^{1}, I\right)$.

Proof. The proof is left to the reader.

Define the following abelian group:

$$
\mathcal{J}_{I}^{1}(E)=\mathcal{J}_{I}^{1} \otimes_{B} E \cong I \otimes_{B} E \oplus E .
$$

Define the following left and right action of $\mathcal{J}_{I}^{1}$ on $\mathcal{J}_{I}^{1}(E)$ :

$$
(x, a)(z \otimes e, f)=(x \otimes f+a z \otimes e+d(a) \otimes f, a f)
$$

and

$$
(z \otimes e, f)(x, a)=(z \otimes e a, f a)
$$

Note: We may write

$$
(x, a)(z \otimes e, f)=\left((a z) \otimes e+d_{\mathcal{J}}(x, a) \otimes f, a f\right) .
$$


Definition 2.3. Let $\mathcal{J}_{I}^{1}(E)$ with the actions (2.2.1) and (2.2.2) be the first $I$-jet module of $E$ with respect to $d$.

There is an exact sequence of abelian groups

$$
0 \rightarrow I \otimes_{B} E \rightarrow \mathcal{J}_{I}^{1}(E) \rightarrow E \rightarrow 0 .
$$

There is a left and right action of $\mathcal{J}_{I}^{1}$ on $E$ defined as follows:

$$
\begin{aligned}
& (x, a) e=a e, \\
& e(x, a)=e a .
\end{aligned}
$$

Proposition 2.4. The actions (2.2.1) and (2.2.2) make $\mathcal{J}_{I}^{1}(E)$ into a left and right $\mathcal{J}_{I}^{1}$-module. The exact sequence (2.3.1) is an exact sequence of left and right $\mathcal{J}_{I}^{1}$-modules.

Proof. The proof is left to the reader.

Corollary 2.5. The sequence (2.3.1) is an exact sequence of left and right Bmodules. It is split exact as right $B$-modules.

Proof. Use the ring homomorphism $t: B \rightarrow \mathcal{J}_{I}^{1}$ defined by $t(b)=(0, b)$. It follows that the maps in the sequence (2.3.1) are $B$-linear. The claim now follows from Proposition 2.4.

Definition 2.6. Let the sequence (2.3.1) be the Atiyah-Karoubi sequence of $E$ with respect to the pair $\left(I, d_{\mathcal{J}}\right)$.

Note: The exact sequence (2.3.1) was first defined in 2 in the case when $\mathcal{I}=$ $\Omega_{X / \mathbf{C}}, d$ is the universal derivation and $X$ is a complex manifold.

Recall the following definition: Given two left $B$-modules $E, F$ we define

$$
\operatorname{Diff}_{A}^{0}(E, F)=\operatorname{Hom}_{B}(E, F)
$$

and

$$
\operatorname{Diff}_{A}^{k}(E, F)=\left\{\partial \in \operatorname{Hom}_{A}(E, F):[\partial, a] \in \operatorname{Diff}_{A}^{k-1}(E, F) \text { for all } a \in B\right\} .
$$

There is a natural map

$$
d_{E}: E \rightarrow \mathcal{J}_{I}^{1}(E)
$$

defined by

$$
d_{E}(e)=(0,1) \otimes e
$$

called the universal differential operator of $E$ with respect to the pair $(I, d)$. Here $(0,1)=\mathbf{1} \in \mathcal{J}_{I}^{1}=I \oplus B$ is the multiplicative unit.

Lemma 2.7. It follows that $d_{E} \in \operatorname{Diff}_{A}^{1}\left(E, \mathcal{J}_{I}^{1}(E)\right)$.

Proof. Recall the following: For elements $(x, a) \in \mathcal{J}_{I}^{1}$ and $b \in B$ it follows that

$$
(x, a)_{t} b=(x, a)(0, b)=(x b, a b)
$$

and

$$
b_{s}(x, a)=(d(b), b)(x, a)=(b x+d(b) a, b a) .
$$


It follows that $d_{E} \in \operatorname{Diff}^{1}\left(E, \mathcal{J}_{I}^{1}(E)\right)$ if and only if $\left[d_{E}, a\right] \in \operatorname{Hom}_{B}\left(E, \mathcal{J}_{I}^{1}(E)\right)$ for all $a \in B$. We get

$$
\begin{gathered}
{\left[d_{E}, a\right](b e)=\left(d_{E} a-a d_{E}\right)(b e)=d_{E}(a b e)-a d_{E}(b e)=(0,1) \otimes a b e-a(0,1) \otimes b e} \\
=(0, a b) \otimes e-a(0,1) \otimes b e=(0, a b) \otimes e-(d(a) b, a b) \otimes e \\
=(-d(a) b, 0) \otimes e .
\end{gathered}
$$

By definition

$$
a(0,1)=(d(a), a)=(d(a), 0)+(0, a)=(d(a), 0)+(0,1) a .
$$

It follows that

$$
(-d(a), 0)=(0,1) a-a(0,1)
$$

We get

$$
\begin{gathered}
\left.\left[d_{E}, a\right](b e)=(-d(a) b, 0) \otimes e=b(-d(a), 0) \otimes e\right)=b((0,1) a \otimes e-a(0,1) \otimes e) \\
=b((0,1) \otimes a e-a(0,1) \otimes e)=b\left(d_{E}(a e)-a d_{E}(e)\right)=b\left[d_{E}, a\right](e) .
\end{gathered}
$$

Hence $\left[d_{E}, a\right] \in \operatorname{Hom}_{B}\left(E, \mathcal{J}_{I}^{1}(E)\right)=\operatorname{Diff}^{0}\left(E, \mathcal{J}_{I}^{1}(E)\right)$ for all $a \in B$. It follows that $d_{E} \in \operatorname{Diff}_{A}^{1}\left(E, \mathcal{J}_{I}^{1}(E)\right)$ and the claim follows.

Recall there is a derivation $d_{\mathcal{J}} \in \operatorname{Der}_{A}\left(\mathcal{J}_{I}^{1}, I\right)$ defined by $d_{\mathcal{J}}(x, a)=x+d(a)$. It follows that $\left.d_{\mathcal{J}}\right|_{B}=d_{I}$. Let $F$ be a left $\mathcal{J}_{I}^{1}$-module. An $A$-linear map

$$
\nabla: F \rightarrow I \otimes_{B} F
$$

satisfying

$$
\nabla((x, a) f)=(x, a) \nabla(f)+d_{\mathcal{J}}(x, a) \otimes f
$$

is an $\left(I, d_{\mathcal{J}}\right)$-connection on $F$. Assume $E$ is a $B$-module. An $A$-linear map

$$
\nabla: E \rightarrow I \otimes_{B} E
$$

satisfying

$$
\nabla(a e)=a \nabla(e)+d(a) \otimes e
$$

is an $(I, d)$-connection.

Lemma 2.8. Assume $\nabla$ is an $\left(I, d_{\mathcal{J}}\right)$-connection. It follows that $\nabla$ is an $(I, d)$ connection.

Proof. Let $b \in B$ and $f \in F$. We get

$$
\begin{gathered}
\nabla(b f)=\nabla((0, b) f)=(0, b) \nabla(f)+d_{\mathcal{J}}(0, b) \otimes f \\
=b \nabla(f)+d(b) \otimes f
\end{gathered}
$$

and the claim is proved.

The sequence (2.3.1) is an exact sequence of left $\mathcal{J}_{I}^{1}$-modules. We get an extension class

$$
c_{\mathcal{J}}(E) \in \operatorname{Ext}_{\mathcal{J}_{I}^{1}}^{1}\left(E, I \otimes_{B} E\right)
$$

When we restrict to $B$ we get an extension class

$$
c_{B}(E) \in \operatorname{Ext}_{B}^{1}\left(E, I \otimes_{B} E\right) .
$$

Definition 2.9. Let $c_{\mathcal{J}}(E)$ be the Atiyah class of $E$ with respect to $\mathcal{J}_{I}^{1}$. Let $c_{B}(E)$ be the Atiyah class of $E$ with respect to $B$. 
Proposition 2.10. The following hold:

(2.10.1) 2.3.1) is split as a sequence of right $\mathcal{J}_{I}^{1}$-modules.

(2.10.2) 2.3.1) is left split as $\mathcal{J}_{I}^{1}$-modules iff $E$ has an $\left(I, d_{\mathcal{J}}\right)$-connection.

(2.10.3) 2.3.1) is left split as B-modules iff $E$ has an $(I, d)$-connection.

Proof. We prove claim (2.10.1): Define the map

$$
s: E \rightarrow \mathcal{J}_{I}^{1}(E)
$$

by

$$
s(e)=(0, e)
$$

It follows that

$$
s(e(x, a))=s(e a)=(0, e a)=(0, e)(x, a)=s(e)(x, a)
$$

and claim (2.10.1) is proved. We prove claim (2.10.2): Assume $s(e)=(\nabla(e), e)$ is a left $\mathcal{J}_{I}^{1}$-linear section. We get

$$
\begin{aligned}
& s((x, a) e) \\
& \quad=(\nabla((x, a) e),(x, a) e)=(\nabla((x, a) e), a e) \\
& \quad=(x, a)(\nabla(e), e)=(x \otimes e+a \nabla(e)+d(a) \otimes e, a e) \\
& \quad=((x, a) \nabla(e)+(x+d(a)) \otimes e, a e) .
\end{aligned}
$$

It follows that $\nabla$ satisfies

$$
\nabla((x, a) e)=(x, a) \nabla(e)+d_{\mathcal{J}}(x, a) \otimes e
$$

and $\nabla$ is an $\left(I, d_{\mathcal{J}}\right)$-connection. Claim (2.10.2) follows. Claim (2.10.3) follows in a similar way and the proposition is proved.

Example 2.11. The classical Atiyah class.

When $I=\Omega_{B / A}=\Omega$ and $d: B \rightarrow \Omega$ is the universal derivation it follows that the class $c_{B}(E)$ is the classical Atiyah class as defined in [2]. From Proposition 2.10] it follows that $c_{B}(E)=0$ if and only if $E$ has a connection

$$
\nabla: E \rightarrow \Omega_{B / A} \otimes_{B} E .
$$

Corollary 2.12. If $c_{\mathcal{J}}(E)=0$, it follows that $c_{B}(E)=0$.

Proof. If $c_{\mathcal{J}}(E)=0$ it follows from Proposition 2.10, claim (2.10.2), that $E$ has an $\left(I, d_{\mathcal{J}}\right)$-connection. It follows from Lemma 2.8 that $E$ has an $(I, d)$-connection. From this and Proposition 2.10, claim (2.10.3), the corollary follows.

Let $\mathcal{J}_{I}^{1}(E)^{\text {left }}$ denote the abelian group $\mathcal{J}_{I}^{1}(E)$ with its left $B$-module structure. Let $\mathcal{J}_{I}^{1}(E)^{\text {right }}$ denote the abelian group $\mathcal{J}_{I}^{1}(E)$ with its right $B$-module structure. We say that the module $I$ is abelianized if the following holds:

$$
a x=x a
$$

for all $a \in B$ and $x \in I$. Define the following product on $I$ :

$$
a * x=x a
$$

for all $a \in B$ and $x \in I$. It follows that we have defined a $B$-module structure on $I$, denoted $I^{\text {star }}$, with the property that there is an isomorphism

$$
I^{\text {right }} \cong I^{\text {star }}
$$


of $A$-modules. When we form the tensor product

$$
I \otimes_{B} E,
$$

we use the right structure on $I$ and left structure on $E$. Since $B$ is commutative it follows that $E$ has a canonical right $B$-module structure. The abelian group $I \otimes_{B} E$ has a left and right structure as a $B$-module, denoted

$$
I \otimes_{B} E^{l e f t}
$$

and

$$
I \otimes_{B} E^{\text {right }} .
$$

We form a new product on $I \otimes_{B} E$ as follows:

$$
a *(x \otimes e)=x \otimes(e a)
$$

for any $a \in B$ and $x \otimes e \in I \otimes_{B} E$. We get a left $B$-module denoted $I \otimes_{B} E^{\text {star }}$. There is an isomorphism

$$
I \otimes_{B} E^{\text {star }} \cong I \otimes_{B} E^{r i g h t}
$$

of $B$-modules.

Lemma 2.13. Assume $I$ is abelianized. It follows that there is an isomorphism

$$
I \otimes_{B} E^{l e f t} \cong I \otimes_{B} E^{\text {right }}
$$

of B-modules.

Proof. Define the map

$$
\phi: I \otimes_{B} E^{l e f t} \rightarrow I \otimes_{B} E^{s t a r}
$$

by

We get

$$
\phi(x \otimes e)=x \otimes e .
$$

$$
\begin{gathered}
\phi(a(x \otimes e))=\phi((a x) \otimes e)=(a x) \otimes e=(x a) \otimes e \\
=x \otimes(a e)=x \otimes(e a)=a *(x \otimes e)=a * \phi(x \otimes e) .
\end{gathered}
$$

It follows that

$$
I \otimes_{B} E^{l e f t} \cong I \otimes_{B} E^{\text {star }} \cong I \otimes_{B} E^{\text {right }}
$$

and the lemma follows.

Proposition 2.14. Assume $I$ is abelianized. The following hold:

(2.14.1) $\quad c_{B}(E)=0$ iff $\mathcal{J}_{I}^{1}(E)^{\text {left }} \cong \mathcal{J}_{I}^{1}(E)^{\text {right }}$ as B-modules.

(2.14.2) $\mathcal{J}_{I}^{1}(E)^{\text {left }} \cong \mathcal{J}_{I}^{1}(E)^{\text {right }}$ as abelian groups.

(2.14.3) $\mathcal{J}_{I}^{1}(E)^{\text {left }} \cong \mathcal{J}_{I}^{1}(E)^{\text {right }}$ as B-modules iff $E$ has an $(I, d)$-connection.

Proof. It follows that $c_{B}(E)=0$ if and only if the sequence

$$
0 \rightarrow I \otimes_{B} E \rightarrow \mathcal{J}_{I}^{1}(E) \rightarrow E \rightarrow 0
$$

is split as a sequence of left $B$-modules. From Lemma 2.13 it follows that

$$
\begin{aligned}
& \mathcal{J}_{I}^{1}(E)^{\text {left }} \cong I \otimes_{B} E \oplus E^{\text {left }} \\
\cong & I \otimes_{A} E \oplus E^{\text {right }} \cong \mathcal{J}_{I}^{1}(E)^{\text {right }}
\end{aligned}
$$


since by Proposition 2.10, claim (2.10.1), the sequence is always split as right $B$ modules. Claim (2.14.1) follows. Claim (2.14.2) is obvious. Claim (2.14.3): From (2.14.1) we get

$$
\mathcal{J}_{I}^{1}(E)^{l e f t} \cong \mathcal{J}_{I}^{1}(E)^{\text {right }}
$$

as $B$-modules iff $c_{B}(E)=0$. From Proposition 2.10, claim (2.10.3), it follows that $c_{B}(E)=0$ iff $E$ has an $(I, d)$-connection. Claim (2.14.3) is proved and the proposition follows.

Hence the characteristic class $c_{B}(E)$ measures when the abelian group $\mathcal{J}_{I}^{1}(E)$ may be equipped with two nonisomorphic structures as a $B$-module.

\section{Generalized Atiyah Classes for quasi-COHerent sheaves}

In this section we generalize the results in the previous section to the case where we consider an arbitrary morphism $\pi: X \rightarrow S$ of schemes and an arbitrary quasicoherent $\mathcal{O}_{X}$-module $\mathcal{E}$. We define the generalized Atiyah class $c_{\mathcal{J}}(\mathcal{E})$ and $c_{\mathcal{O}_{X}}(\mathcal{E})$ using derivations and sheaves of associative rings and prove various properties of this construction. We end the section with a discussion of explicit examples. We give examples of jet bundles $\mathcal{J}_{\Omega}^{1}(\mathcal{O}(d))$, where $\mathcal{O}(d)=\mathcal{O}(1)^{\otimes d}$ and $\mathcal{O}(1)$ is the tautological quotient bundle on $\mathbf{P}_{K}^{1}$. We prove $c_{\mathcal{O}_{\mathbf{P}^{1}}}(\mathcal{O}(d)) \neq 0$ when $\operatorname{char}(K)=0$ and $c_{\mathcal{O}_{\mathbf{P}^{1}}}(\mathcal{O}(d))=0$ when $\operatorname{char}(K)$ divides $d$. Hence the characteristic classes $c_{\mathcal{J}}$ and $c_{\mathcal{O}_{X}}$ are nontrivial.

Let in the following $\pi: X \rightarrow S$ be an arbitrary morphism of schemes and let $\mathcal{I}$ be an arbitrary quasi-coherent left and right $\mathcal{O}_{X}$-module. Let

$$
d \in \operatorname{Der}_{\pi^{-1}\left(\mathcal{O}_{S}\right)}\left(\mathcal{O}_{X}, \mathcal{I}\right)
$$

be a derivation. We may form the quasi-coherent sheaf $\mathcal{J}_{\mathcal{I}}^{1}=\mathcal{I} \oplus \mathcal{O}_{X}$. Let $V \subseteq U$ be open subsets of $X$ and let $(x, a),(y, b) \in \mathcal{J}_{\mathcal{I}}^{1}(U)$ be two elements. Define

$$
(x, a)(y, b)=(x b+a y, a b) .
$$

It follows that $\mathcal{J}_{\mathcal{I}}^{1}(U)$ is an associative ring with multiplicative unit $\mathbf{1}=(0,1)$ and the restriction morphism

$$
\mathcal{J}_{\mathcal{I}}^{1}(U) \rightarrow \mathcal{J}_{\mathcal{I}}^{1}(V)
$$

is a morphism of unital rings. There is a natural embedding

$$
i: \mathcal{I} \rightarrow \mathcal{J}_{\mathcal{I}}^{1}
$$

defined as follows:

$$
\begin{gathered}
i(U): \mathcal{I}(U) \rightarrow \mathcal{J}_{\mathcal{I}}^{1}(U), \\
i(U)(x)=(x, 0) .
\end{gathered}
$$

It follows that $\mathcal{I} \subseteq \mathcal{J}_{\mathcal{I}}^{1}$ is a sheaf of ideals in $\mathcal{J}_{\mathcal{I}}^{1}$ with $\mathcal{I}^{2}=0$. We get an exact sequence

$$
0 \rightarrow \mathcal{I} \rightarrow \mathcal{J}_{\mathcal{I}}^{1} \rightarrow \mathcal{O}_{X} \rightarrow 0
$$

of sheaves of abelian groups. The sequence (3.0.4) is an extension of $\mathcal{O}_{X}$ by a quasi-coherent sheaf of two-sided ideals of square zero. 
We may form the quasi-coherent sheaf $\mathcal{J}_{\mathcal{I}}^{1}(\mathcal{E})=\mathcal{I} \otimes_{\mathcal{O}_{X}} \mathcal{E} \oplus \mathcal{E}$. We may define a left and right $\mathcal{O}_{X}$-structure and a left $\mathcal{J}_{\mathcal{I}}^{1}$-module structure on $\mathcal{J}_{\mathcal{I}}^{1}(\mathcal{E})$ as follows: Let $(x, a) \in \mathcal{J}_{\mathcal{I}}^{1}(U)$ and $(z \otimes e, f) \in \mathcal{J}_{\mathcal{I}}^{1}(\mathcal{E})(U)$. Let

$$
(x, a)(z \otimes e, f)=(x \otimes f+a z \otimes e+d(a) \otimes f, a f)
$$

and

$$
(z \otimes e, f)(x, a)=(z \otimes e a, f a) .
$$

One checks that for any open sets $V \subseteq U$ the restriction morphism

$$
\rho_{U V}: \mathcal{J}_{\mathcal{I}}^{1}(\mathcal{E})(U) \rightarrow \mathcal{J}_{\mathcal{I}}^{1}(\mathcal{E})(V)
$$

satisfies

$$
\left.(x, a)(z \otimes e, f)\right|_{V}=\left.\left.(x, a)\right|_{V}(z \otimes e, f)\right|_{V} .
$$

For open sets $W \subseteq V \subseteq U$ it follows that

$$
\rho_{V W} \circ \rho_{U V}=\rho_{U W} .
$$

Similar formulas hold for the right structure as a $\mathcal{J}_{\mathcal{I}}^{1}$-module. It follows that $\mathcal{J}_{\mathcal{I}}^{1}(\mathcal{E})$ becomes a sheaf of left and right $\mathcal{J}_{\mathcal{I}}^{1}$-modules and left and right $\mathcal{O}_{X}$-modules. We get an exact sequence

$$
0 \rightarrow \mathcal{I} \otimes_{\mathcal{O}} \mathcal{E} \rightarrow \mathcal{J}_{\mathcal{I}}^{1}(\mathcal{E}) \rightarrow \mathcal{E} \rightarrow 0
$$

of sheaves of left and right $\mathcal{O}_{X}$-modules and left and right $\mathcal{J}_{\mathcal{I}}^{1}$-modules. These assertions follow immediately from the local situation since all sheaves involved are quasi-coherent.

Definition 3.1. Let the sequence (3.0.5) be the Atiyah-Karoubi sequence of $\mathcal{E}$ with respect to $(\mathcal{I}, d)$.

Define the $\pi^{-1}\left(\mathcal{O}_{S}\right)$-linear map

$$
d_{\mathcal{E}}: \mathcal{E} \rightarrow \mathcal{J}_{\mathcal{I}}^{1}(\mathcal{E})
$$

by

$$
d_{\mathcal{E}}(U)(e)=(0,1) \otimes e \in \mathcal{J}_{\mathcal{I}}^{1}(\mathcal{E})(U) .
$$

Here $U \subseteq X$ is an open subset.

Lemma 3.2. The following holds:

$$
d_{\mathcal{E}} \in \operatorname{Diff}_{\pi^{-1}\left(\mathcal{O}_{S}\right)}^{1}\left(\mathcal{E}, \mathcal{J}_{\mathcal{I}}^{1}(\mathcal{E})\right) .
$$

Proof. The claim of the lemma follows from Lemma 2.7 since the sheaf $\mathcal{E}$ is quasicoherent.

The morphism $d_{\mathcal{E}}$ is the universal differential operator for $\mathcal{E}$ with respect to $(\mathcal{I}, d)$.

Example 3.3. The first order jet bundle.

Assume $\mathcal{I}=\Omega_{X / S}^{1}$ and $d$ the universal derivation. We get a map

$$
d_{\mathcal{E}}: \mathcal{E} \rightarrow \mathcal{J}_{X / S}^{1}(\mathcal{E})
$$

defined by

$$
d_{\mathcal{E}}(U)(e)=1 \otimes e \in \mathcal{J}_{X / S}^{1}(\mathcal{E})(U) .
$$

This map is the classical differential operator $d_{\mathcal{E}} \in \operatorname{Diff}_{\pi^{-1}\left(\mathcal{O}_{S}\right)}^{1}\left(\mathcal{E}, \mathcal{J}_{X / S}^{1}(\mathcal{E})\right)$ for $\mathcal{E}$. 
In the following we view the sequence (3.0.5) as an exact sequence of sheaves of left $\mathcal{J}_{\mathcal{I}}^{1}$ and $\mathcal{O}_{X}$-modules. We get a characteristic class

$$
c_{\mathcal{J}}(\mathcal{E}) \in \operatorname{Ext}_{\mathcal{J}_{\mathcal{I}}^{1}}^{1}\left(\mathcal{E}, \mathcal{I} \otimes_{\mathcal{O}_{X}} \mathcal{E}\right)
$$

and

$$
c_{\mathcal{O}_{X}}(\mathcal{E}) \in \operatorname{Ext}_{\mathcal{O}_{X}}^{1}\left(\mathcal{E}, \mathcal{I} \otimes_{\mathcal{O}_{X}} \mathcal{E}\right) .
$$

Definition 3.4. Let $c_{\mathcal{J}}(\mathcal{E})$ be the Atiyah class of $\mathcal{E}$ with respect to $\mathcal{J}_{\mathcal{I}}^{1}$. Let $c_{\mathcal{O}_{X}}(\mathcal{E})$ be the Atiyah class of $\mathcal{E}$ with respect to $\mathcal{O}_{X}$.

There is a derivation $d_{\mathcal{J}} \in \operatorname{Der}_{\pi^{-1}\left(\mathcal{O}_{S}\right)}\left(\mathcal{J}_{\mathcal{I}}^{1}, \mathcal{I}\right)$ defined as follows: Let $(x, a) \in$ $\mathcal{J}_{\mathcal{I}}^{1}(U)$. Define

$$
d_{\mathcal{J}}(x, a)=x+d(a) .
$$

Assume $\mathcal{E}$ is a quasi-coherent $\mathcal{O}_{X}$-module. We say that a $\pi^{-1}\left(\mathcal{O}_{S}\right)$-linear map

$$
\nabla: \mathcal{E} \rightarrow \mathcal{I} \otimes_{\mathcal{O}_{X}} E
$$

is an $(\mathcal{I}, d)$-connection if for all local sections $a \in \mathcal{O}(U)$ and $e \in \mathcal{E}(U)$ on an open set $U \subseteq X$ the following holds:

$$
\nabla(a e)=a \nabla(e)+d(a) \otimes e .
$$

Assume $\mathcal{F}$ is a left $\mathcal{J}_{\mathcal{I}}^{1}$-module which is quasi-coherent as a left $\mathcal{O}_{X}$-module. We say that a $\pi^{-1}\left(\mathcal{O}_{S}\right)$-linear map

$$
\nabla: \mathcal{F} \rightarrow \mathcal{I} \otimes \mathcal{O}_{X} \mathcal{F}
$$

is an $\left(\mathcal{I}, d_{\mathcal{J}}\right)$-connection if the following holds for $(x, a) \in \mathcal{J}_{\mathcal{I}}^{1}(U)$ and $f \in \mathcal{F}(U)$ :

$$
\nabla((x, a) f)=(x, a) \nabla(f)+d_{\mathcal{J}}(x, a) \otimes f .
$$

Proposition 3.5. The following hold:

$$
\begin{aligned}
& c_{\mathcal{J}}(\mathcal{E})=0 \text { iff } \mathcal{E} \text { has an }\left(\mathcal{I}, d_{\mathcal{J}}\right) \text {-connection. } \\
& c_{\mathcal{O}_{X}}(\mathcal{E})=0 \text { iff } \mathcal{E} \text { has an }(\mathcal{I}, d) \text {-connection. } \\
& \text { If } c_{\mathcal{J}}(\mathcal{E})=0 \text {, it follows that } c_{\mathcal{O}_{X}}(\mathcal{E})=0 .
\end{aligned}
$$

Proof. The proof is left to the reader.

We say that the sheaf $\mathcal{I}$ is abelianized if for all local sections $a$ of $\mathcal{O}_{X}$ and $x$ of $\mathcal{I}$ the following holds:

$$
a x=x a .
$$

As in Lemma 2.13, we get an isomorphism

$$
I \otimes \mathcal{O}_{X} \mathcal{E} \oplus \mathcal{E}^{\text {left }} \cong I \otimes_{\mathcal{O}_{X}} \mathcal{E} \oplus \mathcal{E}^{\text {right }}
$$

of $\mathcal{O}_{X}$-modules.

Theorem 3.6. Assume $\mathcal{I}$ is abelianized. The following hold:

(3.6.1) The sequence (3.0.5) is split as a sequence of right $\mathcal{O}_{X}$-modules.

(3.6.2) 3.0.5 is split as a left $\mathcal{J}_{\mathcal{I}}^{1}$-module iff $\mathcal{E}$ has an $\left(\mathcal{I}, d_{\mathcal{J}}\right)$-connection.

(3.6.3) $\mathcal{J}_{\mathcal{I}}^{1}(\mathcal{E})^{\text {left }} \cong \mathcal{J}_{\mathcal{I}}^{1}(\mathcal{E})^{\text {right }}$ as sheaves of abelian groups.

(3.6.4) $\quad c_{\mathcal{O}_{X}}(\mathcal{E})=0$ iff $\mathcal{J}_{\mathcal{I}}^{1}(\mathcal{E})^{\text {left }} \cong \mathcal{J}_{\mathcal{I}}^{1}(\mathcal{E})^{\text {right }}$ as $\mathcal{O}_{X}$-modules.

(3.6.5) 3.0.5 is split as left $\mathcal{O}_{X}$-modules iff $\mathcal{E}$ has an $(\mathcal{I}, d)$-connection. 
Proof. We prove (3.6.1): Let $U \subseteq X$ be an open subset and define the morphism

$$
s(U): \mathcal{E}(U) \rightarrow \mathcal{J}_{\mathcal{I}}^{1}(\mathcal{E})(U)
$$

by

$$
s(U)(e)=(0, e) .
$$

Let $X=(x \otimes a, b) \in \mathcal{J}_{\mathcal{I}}^{1}(U)$. It follows that

$$
s(U)(e X)=(0, e b)=(0, e) X=s(U)(e) X ;
$$

hence $s$ is left $\mathcal{J}_{\mathcal{I}}^{1}$-linear. The map $s$ splits (3.0.5) as a sequence of left $\mathcal{J}_{\mathcal{I}}^{1}$-modules and claim (3.6.1) follows. We prove (3.6.2): By definition, (3.0.5) is split as left $\mathcal{J}_{\mathcal{I}}^{1}$ modules iff $c_{\mathcal{J}}(\mathcal{E})=0$. By Proposition 3.5 this is iff $\mathcal{E}$ has an $\left(\mathcal{I}, d_{\mathcal{J}}\right)$-connection. Claim (3.6.2) is proved. We prove (3.6.5): The sequence (3.0.5) is split as left $\mathcal{O}_{X}$-modules iff $c_{\mathcal{O}_{X}}(\mathcal{E})=0$. By Proposition 3.5 this occurs iff $\mathcal{E}$ has an $(\mathcal{I}, d)$ connection. Claim (3.6.5) is proved. We prove (3.6.4): By definition it follows that

$$
\mathcal{J}_{\mathcal{I}}^{1}(\mathcal{E})^{\text {right }} \cong \mathcal{I} \otimes_{\mathcal{O}_{X}} \mathcal{E} \oplus \mathcal{E}^{\text {right }}
$$

as right $\mathcal{O}_{X}$-modules. Sequence (3.0.5) is left split as $\mathcal{O}_{X}$-modules iff

$$
\mathcal{J}_{\mathcal{I}}^{1}(\mathcal{E})^{l e f t} \cong \mathcal{I} \otimes_{\mathcal{O}_{X}} \mathcal{E} \oplus \mathcal{E}^{l e f t} .
$$

It follows that $c_{\mathcal{O}_{X}}(\mathcal{E})=0$ iff there is an isomorphism

$$
\mathcal{J}_{\mathcal{I}}^{1}(\mathcal{E})^{l e f t} \cong \mathcal{I} \otimes_{\mathcal{O}_{X}} \mathcal{E} \oplus \mathcal{E}^{\text {left }} \cong \mathcal{I} \otimes_{\mathcal{O}_{X}} \mathcal{E} \oplus \mathcal{E}^{\text {right }} \cong \mathcal{J}_{\mathcal{I}}^{1}(\mathcal{E})^{\text {right }} .
$$

Claim (3.6.4) follows. Claim (3.6.3) is obvious and the theorem follows.

Hence the characteristic class $c_{\mathcal{O}_{X}}(\mathcal{E})$ measures when the sheaf of abelian groups $\mathcal{J}_{\mathcal{I}}^{1}(\mathcal{E})$ is equipped with two nonisomorphic structures as an $\mathcal{O}_{X}$-module.

Example 3.7. The classical case: $\mathcal{I}=\Omega_{X / S}^{1}$.

Assume in the following proposition that $\pi: X \rightarrow S$ is a separated morphism. Let $\Delta: X \rightarrow X \times{ }_{S} X$ be the diagonal embedding. It follows that $\Delta(X) \subseteq X \times{ }_{S}$ $X$ is a closed subscheme. Let $\mathcal{J} \subseteq \mathcal{O}_{X{ }_{\times S} X}$ be the ideal sheaf of $\Delta(X)$ and let $\mathcal{O}_{\Delta^{l}}=\mathcal{O}_{X \times_{S} X} / \mathcal{J}^{l+1}$ be the l-th infinitesimal neighborhood of the diagonal. Let $p, q: X \times_{S} X \rightarrow X$ be the canonical projection maps.

Definition 3.8. Let $\mathcal{J}_{X / S}^{l}(\mathcal{E})=p_{*}\left(\mathcal{O}_{\Delta^{l+1}} \otimes q^{*} \mathcal{E}\right)$ be the l-th order jet bundle of $\mathcal{E}$.

Assume $\pi$ is given by a homomorphism $\phi: A \rightarrow B$ of commutative rings. Let $X=\operatorname{Spec}(B)$ and $S=\operatorname{Spec}(A)$. Assume $\mathcal{E}$ is the sheaffification of a $B$-module $E$. It follows that $\mathcal{J}_{X / S}^{1}(\mathcal{E})$ is the sheaf associated to $P_{B / A}^{l}(E)=B \otimes_{A} B / J^{l+1} \otimes_{B} E$, where $J \subseteq B \otimes_{A} B$ is the kernel of the multiplication map.

Proposition 3.9. Assume $\mathcal{I}=\Omega_{X / S}$ and $d: \mathcal{O}_{X} \rightarrow \Omega_{X / S}$ is the universal derivation. It follows that $\mathcal{J}_{\mathcal{I}}^{1}(\mathcal{E}) \cong \mathcal{J}_{X / S}^{1}(\mathcal{E})$ is the first order jet bundle of $\mathcal{E}$. The generalized Atiyah sequence (3.0.5) becomes the classical Atiyah sequence.

Proof. Assume $U=\operatorname{Spec}(B) \subseteq X$ is an open affine subscheme mapping to an open affine subscheme $V=\operatorname{Spec}(A) \subseteq S$. Let $\mathcal{E}(U)=E$, where $E$ is a $B$-module and let $\Omega_{X / S} \mid(U)=\Omega$. Let $m: B \otimes_{A} B \rightarrow B$ be the multiplication map and let $s: B \rightarrow P_{B / A}^{1}$ be defined by $s(b)=1 \otimes b$. It follows that there is an isomorphism

$$
\phi: P_{B / A}^{1} \otimes_{B} E \rightarrow \Omega \otimes_{B} E \oplus E
$$


defined by

$$
\phi(x \otimes e)=((x-s m(x)) \otimes e, m(x) e) .
$$

One checks that we get a commutative diagram of exact sequences

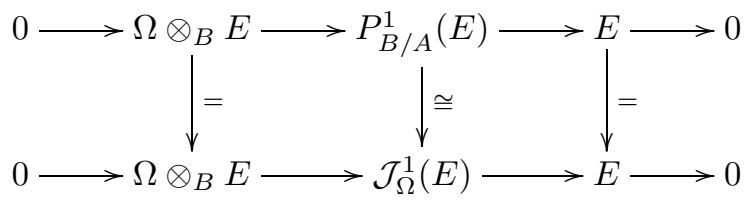

where the middle vertical arrow is the isomorphism $\phi$. Since the map $\phi$ is intrinsically defined it glues to give an isomorphism $\mathcal{J}_{X / S}^{1}(\mathcal{E}) \cong \mathcal{J}_{\Omega}^{1}(\mathcal{E})$. The proposition follows.

Note: If $I=\Omega_{B / A}$ and $d: B \rightarrow \Omega_{B / A}$ is the universal derivation the construction of $\mathcal{J}_{\Omega_{B / A}}^{1}(E)$ using $d$ is due to Karoubi (see [6]). In this case we get the sequence

$$
0 \rightarrow \Omega_{B / A} \otimes_{B} E \rightarrow P_{B / A}^{1}(E) \rightarrow E \rightarrow 0,
$$

where $P_{B / A}^{1}(E)$ is the first order jet module of $E$. It is also called the first order module of principal parts of $E$.

Note: Assume $\mathcal{E}$ is a finite rank locally free $\mathcal{O}_{X}$-module. If $X$ is a smooth scheme of finite type over $\mathbf{C}$, the complex numbers, $\mathcal{I}=\Omega_{X}^{1}$ is the module of differentials and $d$ the universal derivation we get the classical Atiyah sequence

$$
0 \rightarrow \Omega_{X}^{1} \otimes \mathcal{E} \rightarrow \mathcal{J}_{\Omega_{X}^{1}}^{1}(\mathcal{E}) \rightarrow \mathcal{E} \rightarrow 0
$$

The class

$$
c_{\mathcal{O}_{X}}(\mathcal{E}) \in \operatorname{Ext}_{\mathcal{O}_{X}}^{1}\left(\mathcal{E}, \Omega_{X}^{1} \otimes \mathcal{E}\right)
$$

is the classical Atiyah class as defined in [2]. It follows that $c_{\mathcal{O}_{X}}(\mathcal{E})=0$ iff $\mathcal{E}$ has a connection

$$
\nabla: \mathcal{E} \rightarrow \Omega_{X}^{1} \otimes \mathcal{E}
$$

Example 3.10. Sheaves of jets on the projective line.

Let $K$ be a field of characteristic zero and consider $\mathbf{P}_{K}^{1}$. Let $\mathcal{O}(d)$ be $\mathcal{O}(1)^{\otimes d}$, where $\mathcal{O}(1)$ is the tautological quotient bundle on $\mathbf{P}_{K}^{1}$. Let $\Omega=\Omega_{\mathbf{P}_{K}^{1}}^{1}$ be the sheaf of differentials on $\mathbf{P}_{K}^{1}$. We get from Proposition 3.9 an exact sequence of $\mathcal{O}_{\mathbf{P}^{1} \text {-modules }}$

$$
0 \rightarrow \Omega \otimes \mathcal{O}(d) \rightarrow \mathcal{J}_{\Omega}^{1}(\mathcal{O}(d)) \rightarrow \mathcal{O}(d) \rightarrow 0
$$

It follows that $\mathcal{J}_{\Omega}^{1}(\mathcal{O}(d)) \cong \mathcal{J}_{\mathbf{P}^{1}}^{1}(\mathcal{O}(d))$ is the first order jet bundle of $\mathcal{O}(d)$ on

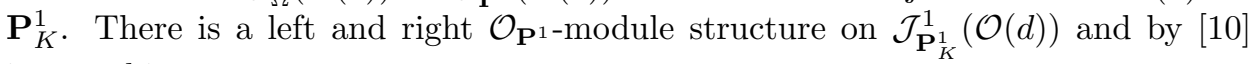
isomorphisms

$$
\mathcal{J}_{\mathbf{P}_{K}^{1}}^{1}(\mathcal{O}(d))^{l e f t} \cong \mathcal{O}(d-1) \oplus \mathcal{O}(d-1)
$$

and

$$
\mathcal{J}_{\mathbf{P}_{K}^{1}}^{1}(\mathcal{O}(d))^{\text {right }} \cong \mathcal{O}(d) \oplus \mathcal{O}(d-2)
$$

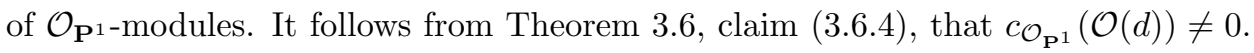
There is an isomorphism

$$
\mathcal{J}_{\mathbf{P}_{K}^{1}}^{1}(\mathcal{O}(d))^{l e f t} \cong \mathcal{J}_{\mathbf{P}_{K}^{1}}^{1}(\mathcal{O}(d))^{r i g h t}
$$

as sheaves of abelian groups. 
By Proposition 3.5 it follows that $c_{\mathcal{J}}(\mathcal{O}(d)) \neq 0$ if $\operatorname{char}(K)=0$; hence the classes $c_{\mathcal{O}_{\mathrm{P}^{1}}}(\mathcal{O}(d))$ and $c_{\mathcal{J}}(\mathcal{O}(d))$ are nontrivial.

Again by [10] if $\operatorname{char}(K)$ divides $d$ it follows that there is an isomorphism

$$
\mathcal{J}_{\mathbf{P}_{K}^{1}}^{1}(\mathcal{O}(d))^{\text {left }} \cong \mathcal{J}_{\mathbf{P}_{K}^{1}}^{1}(\mathcal{O}(d))^{\text {right }}
$$

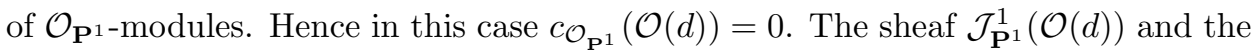
class $c_{\mathcal{O}_{\mathbf{P}^{1}}}(\mathcal{O}(d))$ are defined over $\mathbf{P}_{\mathbf{Z}}^{1}$. When we pass to $\mathbf{Q}$ the class $c_{\mathcal{O}_{\mathbf{P}^{1}}}(\mathcal{O}(d))$ is nonzero. When we pass to $\mathbf{F}_{p}=\mathbf{Z} / p \mathbf{Z}$ and when $p$ divides $d$ the class $c_{\mathcal{O}_{\mathbf{P} 1}}(\mathcal{O}(d))$ becomes zero.

\section{Jets AND InFINITESimal EXTENSIONS OF SHEAVES}

In this section we define and study jet bundles $\mathcal{J}_{\mathcal{I}}^{1}(\mathcal{E})$ of $\mathcal{O}_{X}$-modules $\mathcal{E}$ relative to any morphism $\pi: X \rightarrow S$ of schemes. We define in Definition 4.2 the generalized Atiyah sequence of $\mathcal{E}$ with respect to a square zero extension $\mathcal{J}$ and a derivation $d \in \operatorname{Der}_{\mathcal{R}}\left(\mathcal{O}_{X}, \mathcal{I}\right)$. Here $\mathcal{J}$ is any sheaf of associative unital algebras on $X$ that are square zero extensions of $\mathcal{O}_{X}$ with respect to a quasi-coherent left and right $\mathcal{O}_{X^{-}}$ module $\mathcal{I}$. We use the generalized Atiyah sequence of Definition 4.2 in Definition 4.4 to define the generalized Atiyah classes $c_{\mathcal{J}}(\mathcal{E})$ and $c_{\mathcal{O}_{X}}(\mathcal{E})$ for $\mathcal{E}$. This construction generalizes the classical construction (see Example 4.5).

In the following let $\pi: X \rightarrow S$ be a morphism of schemes and let $\mathcal{R}=\pi^{-1}\left(\mathcal{O}_{S}\right)$. It follows that $\mathcal{O}_{X}$ is a sheaf of $\mathcal{R}$-algebras. This means for any open subset $U \subseteq X$ that the ring $\mathcal{O}_{X}(U)$ is an $\mathcal{R}(U)$-algebra. Let $\mathcal{E}$ be a quasi-coherent sheaf of left $\mathcal{O}_{X}$-modules. It follows that $\mathcal{E}$ is canonically a sheaf of quasi-coherent right $\mathcal{O}_{X^{-}}$ modules. Let

$$
0 \rightarrow \mathcal{I} \rightarrow \mathcal{J} \rightarrow^{m} \mathcal{O}_{X} \rightarrow 0
$$

be an infinitesimal exstension of $\mathcal{O}_{X}$ by a quasi-coherent left and right $\mathcal{O}_{X}$-module $\mathcal{I}$. This means that $\mathcal{J}$ is a sheaf of associative unital rings on $X$ and $\mathcal{I} \subseteq \mathcal{J}$ is a sheaf of quasi-coherent two-sided ideals with $\mathcal{I}^{2}=0$. Let $d \in \operatorname{Der}_{\mathcal{R}}\left(\mathcal{O}_{X}, \mathcal{I}\right)$ be a derivation over $\mathcal{R}$. This means for any open set $U$ that $d(U) \in \operatorname{Der}_{\mathcal{R}(U)}\left(\mathcal{O}_{X}(U), \mathcal{I}(U)\right)$ is a derivation over $\mathcal{R}(U)$. The derivation $d$ induces a derivation $p=d \circ m$ in $\operatorname{Der}_{\mathcal{R}}(\mathcal{J}, \mathcal{I})$. Make the following definition:

$$
\mathcal{J}_{\mathcal{I}}^{1}(\mathcal{E})=\mathcal{I} \otimes_{\mathcal{O}_{X}} \mathcal{E} \oplus \mathcal{E}
$$

Let $a \in \mathcal{J}(U)$ and $Z=(z \otimes e, f) \in \mathcal{J}_{\mathcal{I}}^{1}(\mathcal{E})(U)$ and define

$$
a Z=a(z \otimes e, f)=((a z) \otimes e+p(a) \otimes f, a f)
$$

and

$$
Z a=(z \otimes e, f) a=(z \otimes(e a), f a) .
$$

This is well defined since the sheaf $\mathcal{O}_{X}$ is a sheaf of commutative rings; hence $\mathcal{E}$ is canonically a sheaf of right $\mathcal{O}_{X}$-modules. It follows for any open subset $V \subseteq U$ that there is an equality

$$
\left.a(z \otimes e, f)\right|_{V}=\left.\left.a\right|_{V}(z \otimes e, f)\right|_{V} .
$$

If $\rho_{U V}$ is the restriction map from

$$
\rho_{U V}: \mathcal{J}_{\mathcal{I}}^{1}(\mathcal{E})(U) \rightarrow \mathcal{J}_{\mathcal{I}}^{1}(\mathcal{E})(V),
$$

it follows for any open sets $W \subseteq V \subseteq U$ that we get

$$
\rho_{V W} \circ \rho_{U V}=\rho_{U W} .
$$


Let $W=(w \otimes g, h) \in \mathcal{J}_{\mathcal{I}}^{1}(\mathcal{E})(U)$ and $b \in \mathcal{J}(U)$.

Proposition 4.1. The sheaf $\mathcal{J}_{\mathcal{I}}^{1}(\mathcal{E})$ is a sheaf of left and right $\mathcal{J}$-modules. The sheaf $\mathcal{I} \otimes_{\mathcal{O}_{X}} \mathcal{E}$ is a sheaf of left and right $\mathcal{J}$-submodules of $\mathcal{J}_{\mathcal{I}}^{1}(\mathcal{E})$. The sheaf $\mathcal{E}$ is a sheaf of left and right $\mathcal{J}$-modules.

Proof. The proof is left to the reader.

Note: $\mathcal{J}_{\mathcal{I}}^{1}(\mathcal{E})$ is not a lifting of $\mathcal{E}$ to $\mathcal{J}$ in the sense of deformation theory since $\mathcal{I} \mathcal{J}_{\mathcal{I}}^{1}(\mathcal{E})=0$. It follows that

$$
\begin{gathered}
\mathcal{O}_{X} \otimes_{\mathcal{J}} \mathcal{J}_{\mathcal{I}}^{1}(\mathcal{E}) \cong(\mathcal{J} / \mathcal{I}) \otimes_{\mathcal{J}} \mathcal{J}_{\mathcal{I}}^{1}(\mathcal{E}) \\
\cong \mathcal{J}_{\mathcal{I}}^{1}(\mathcal{E}) / \mathcal{I} \mathcal{J}_{\mathcal{I}}^{1}(\mathcal{E}) \cong \mathcal{J}_{\mathcal{I}}^{1}(\mathcal{E}) \neq \mathcal{E} .
\end{gathered}
$$

By definition a lifting $\mathcal{E}_{\mathcal{J}}$ of $\mathcal{E}$ to $\mathcal{J}$ is required to satisfy

$$
\mathcal{O}_{X} \otimes_{\mathcal{J}} \mathcal{E}_{\mathcal{J}} \cong \mathcal{E}
$$

We get a natural exact sequence of sheaves of abelian groups

$$
0 \rightarrow \mathcal{I} \otimes \mathcal{O}_{X} \mathcal{E} \rightarrow^{i} \mathcal{J}_{\mathcal{I}}^{1}(\mathcal{E}) \rightarrow^{j} \mathcal{E} \rightarrow 0
$$

Definition 4.2. Let the sequence (4.1.1) be the Atiyah-Karoubi sequence of $\mathcal{E}$ with respect to the pair $(\mathcal{I}, d)$.

Corollary 4.3. The sequence (4.1.1) is an exact sequence of left and right $\mathcal{J}$ modules and left and right $\mathcal{O}_{X}$-modules.

Proof. One checks that the natural maps $i, j$ are left and right $\mathcal{J}$-linear, left and right $\mathcal{O}_{X}$-linear, and the corollary follows.

View the sequence (4.1.1) as a sequence of left $\mathcal{J}$ and $\mathcal{O}_{X}$-modules. We get two characteristic classes,

$$
c_{\mathcal{J}}(\mathcal{E}) \in \operatorname{Ext}_{\mathcal{J}}^{1}\left(\mathcal{E}, \mathcal{I} \otimes_{\mathcal{O}_{X}} \mathcal{E}\right)
$$

and

$$
c_{\mathcal{O}_{X}}(\mathcal{E}) \in \operatorname{Ext}_{\mathcal{O}_{X}}^{1}\left(\mathcal{E}, \mathcal{I} \otimes_{\mathcal{O}_{X}} \mathcal{E}\right) .
$$

Definition 4.4. The class $c_{\mathcal{J}}(\mathcal{E})$ is the Atiyah class of $\mathcal{E}$ with respect to $\mathcal{J}$. The class $c_{\mathcal{O}_{X}}(\mathcal{E})$ is the Atiyah class of $\mathcal{E}$ with respect to $\mathcal{O}_{X}$.

Example 4.5. The classical Atiyah class.

The class $c_{\mathcal{O}_{X}}(\mathcal{E})$ generalizes the classical Atiyah class: When $X$ is a separated scheme over a fixed base scheme $S$ and $\mathcal{I}=\Omega_{X / S}^{1}$ is the sheaf of differentials, $d$ is the universal derivation, $\mathcal{J}=\mathcal{I} \times \mathcal{O}_{X}$ is the trivial square zero extension and $\mathcal{R}=\pi^{-1}\left(\mathcal{O}_{S}\right)$, it follows that $c_{\mathcal{O}_{X}}(\mathcal{E})$ is the classical Atiyah class as defined in [2].

\section{Appendix: Explicit eXAmples}

In this section we give some explicit examples to illustrate the constructions made in the previous sections. In the following let $K$ be an arbitrary field.

Example 5.1. Connections on projective modules. 
Let $A$ be a commutative ring with unit and let $P$ be a finitely generated projective $A$-module. Let

$$
0 \rightarrow K \rightarrow A^{n} \rightarrow^{p} P \rightarrow 0
$$

be an exact sequence, where $A^{n}$ is the free $A$-module of rank $n$. Since $P$ is projective the map $q$ has a section $s$ with $q \circ s=i d$.

Let $F=A^{n}=A\left\{e_{1}, \ldots, e_{n}\right\}$. Let $p_{i}: F \rightarrow A$ be defined by $p_{i}=e_{i}^{*}$. Let $q_{i}=p_{i} \circ s$. We get elements

$$
e_{1}, \ldots, e_{n} \in P
$$

and

$$
q_{1}, \ldots, q_{n} \in P^{*}
$$

satisfying

$$
\sum_{i=1}^{n} q_{i}(a) e_{i}=a
$$

for any $a \in P$. Define the following map:

$$
\nabla(e)=\sum_{i=1}^{n} d\left(q_{i}(e)\right) \otimes e_{i} .
$$

Lemma 5.2. The map $\nabla$ is a connection on $P$.

Proof. The map $\nabla$ is by definition well-defined. We check that it is a connection:

$$
\begin{gathered}
\nabla(a e)=\sum_{i} d\left(q_{i}(a e)\right) \otimes e_{i}=\sum_{i} d\left(a q_{i}(e)\right) \otimes e_{i} \\
=\sum_{i}\left(a d\left(q_{i}(e)\right)+d(a) q_{i}(e)\right) \otimes e_{i}=a \sum_{i} d\left(q_{i}(e)\right) \otimes e_{i}+d(a) \otimes \sum_{i} q_{i}(e) e_{i} \\
=a \nabla(e)+d(a) \otimes e
\end{gathered}
$$

and the map $\nabla$ is a connection. The lemma follows.

The universal derivation $d: A \rightarrow \Omega^{1}$ gives rise to the Atiyah-Karoubi sequence

$$
0 \rightarrow \Omega^{1} \otimes P \rightarrow \mathcal{J}_{\Omega^{1}}^{1}(P) \rightarrow P \rightarrow 0 .
$$

The abelian group $\mathcal{J}^{1}(P)=\mathcal{J}_{\Omega^{1}}^{1}(P)$ has a canonical left $A$-structure and a canonical right $A$-structure. It has by the previous section a nontrivial left $A$-structure defined as follows:

$$
a(x \otimes e, f)=((a x) \otimes e+d(a) \otimes f, a f)
$$

with $a \in A$ and $(x \otimes e, f) \in \mathcal{J}^{1}(P)$. By Lemma 2.8 and Theorem 3.6 there is an isomorphism

$$
\mathcal{J}^{1}(P)^{l e f t} \cong \mathcal{J}^{1}(P)^{\text {right }}
$$

of $A$-modules.

Example 5.3. Generalized Atiyah classes on the projective line.

In this example we consider generalized Atiyah sequences of locally free sheaves on the projective line. We prove that in the case of an invertible sheaf the only non-trivial Atiyah class arises in the classical case. The aim of the constructions made is to construct new examples of locally free sheaves of left and right modules on the projective line where the splitting type as a left module differs from the splitting type as a right module. 
Let $V=K\left\{e_{0}, e_{1}\right\}$ and $V^{*}=K\left\{x_{0}, x_{1}\right\}$ and $\mathbf{P}=\mathbf{P}\left(V^{*}\right)$ be the projective line over $K$. Let $\mathcal{O}(m)=\mathcal{O}(1)^{\otimes m}$ be the $m$-th tensor power of the tautological line bundle on $\mathbf{P}$. There is an isomorphism

$$
\Omega_{\mathbf{P}}^{1} \cong \mathcal{O}(-2),
$$

where $\Omega_{\mathbf{P}}^{1}$ is the cotangent bundle on $\mathbf{P}$. We get an exact sequence

$$
0 \rightarrow \Omega_{\mathbf{P}}^{1} \otimes \mathcal{O}(m) \rightarrow \mathcal{J}_{\mathbf{P}}(\mathcal{O}(m)) \rightarrow \mathcal{O}(m) \rightarrow 0
$$

called the classical Atiyah sequence of $\mathcal{O}(m)$. Assume

$$
d \in \operatorname{Der}_{K}\left(\mathcal{O}_{\mathbf{P}}, \mathcal{O}(m)\right)
$$

is a derivation and consider the generalized Atiyah sequence

$$
0 \rightarrow \mathcal{O}(m) \otimes \mathcal{O}(l) \rightarrow \mathcal{J}_{\mathcal{O}(m)}(\mathcal{O}(l)) \rightarrow \mathcal{O}(l) \rightarrow 0
$$

with respect to $(\mathcal{O}(m), d)$. We get a characteristic class

$$
c_{\mathcal{O}_{\mathbf{P}}}(\mathcal{O}(l)) \in \operatorname{Ext}^{1}(\mathcal{O}(l), \mathcal{O}(m+l)) .
$$

Proposition 5.4. The only nontrivial case is when $m=-2$,

$$
d: \mathcal{O}_{\mathbf{P}} \rightarrow \Omega_{\mathbf{P}}^{1}
$$

is the universal derivation and (5.3.1) is the classical Atiyah sequence.

Proof. The proof is left to the reader.

When we use a derivation $d: \mathcal{O}_{\mathbf{P}} \rightarrow \mathcal{I}$, where $\mathcal{I}$ is a higher rank locally free sheaf we may get new examples.

Let $\mathcal{I}=\mathcal{J}_{\mathbf{P}} \cong \mathcal{O}(-2) \oplus \mathcal{O}$ be the first order jet bundle on $\mathbf{P}$. Define the following derivation $d: \mathcal{O}_{\mathbf{P}} \rightarrow \mathcal{I}$. Let $U_{i}=D\left(x_{i}\right)$ and let $t=x_{1} / x_{0}, s=1 / t$ be local coordinates on $\mathbf{P}$. Let $\mathcal{O}\left(U_{0}\right)=K[t], \mathcal{I}\left(U_{0}\right)=K[t]\{d t, e\}$ and

$$
d_{0}: K[t] \rightarrow K[t]\{d t, e\}
$$

be defined by

$$
d_{0}(a(t))=a^{\prime}(t) d t+t^{i} a^{\prime}(t) e
$$

with $i=0,1,2$. Let $\mathcal{O}\left(U_{1}\right)=K[s], \mathcal{I}\left(U_{1}\right)=K[s]\{d s, f\}$ and

$$
d_{1}: K[s] \rightarrow K[s]\{d s, f\}
$$

be defined by

$$
d_{1}(b(s))=b^{\prime}(s) d s^{-} s^{2-i} b^{\prime}(s) f .
$$

One checks that $d_{0}, d_{1}$ glue to a derivation $d \in \operatorname{Der}\left(\mathcal{O}_{\mathbf{P}}, \mathcal{I}\right)$. We get for any line bundle $\mathcal{O}(l)$ on $\mathbf{P}$ an Atiyah-Karoubi sequence

$$
0 \rightarrow I \otimes \mathcal{O}(l) \rightarrow \mathcal{J}_{\mathcal{I}}(\mathcal{O}(l)) \rightarrow \mathcal{O}(l) \rightarrow 0 .
$$

The local structure of $\mathcal{J}_{\mathcal{I}}(\mathcal{O}(l))$ looks as follows:

$$
\mathcal{J}_{\mathcal{I}}(\mathcal{O}(l))\left(U_{0}\right)=K[t]\left\{d t \otimes x_{0}^{l}, e \otimes x_{0}^{l}\right\}
$$

with left $K[t]$-multiplication given as follows:

$$
a\left(\omega, b x_{0}^{l}\right)=\left(a \omega+d_{0}(a) \otimes b x_{0}^{l}, a b x_{0}^{l}\right) .
$$

Moreover

$$
\mathcal{J}_{\mathcal{I}}(\mathcal{O}(l))\left(U_{1}\right)=K[s]\left\{d s \otimes x_{1}^{l}, f \otimes x_{1}^{l}\right\}
$$


with left $K[s]$-multiplication given as follows:

$$
c\left(\eta, d x_{1}^{l}\right)=\left(c \eta+d_{1}(c) \otimes d x_{1}^{l}, c d x_{1}^{l}\right) .
$$

We aim to construct the structure matrix of the locally free sheaf $\mathcal{J}_{\mathcal{I}}(\mathcal{O}(l))$ as a left $\mathcal{O}_{\mathbf{P}}$-module and to see how this matrix depends on the derivation $d$ and the integer $l$. The splitting type of $\mathcal{J}_{\mathcal{I}}(\mathcal{O}(l))$ as a right $\mathcal{O}_{\mathbf{P}}$-module is by the previous section as follows:

$$
\mathcal{J}_{\mathcal{I}}(\mathcal{O}(l))^{\text {right }} \cong \mathcal{O}(l-2) \oplus \mathcal{O}(l) \oplus \mathcal{O}(l) .
$$

Using Atiyah-Karoubi sequences we hope to give new examples where the splitting type as a left module differs from the one as a right module. Let $\mathcal{J}_{\mathcal{I}}(\mathcal{O}(l))\left(U_{0}\right)$ have the following basis as a left $K[t]$-module:

$$
B:(1,0,0)_{0}=d t \otimes x_{0}^{l},(0,1,0)_{0}=e \otimes x_{0}^{l},(0,0,1)_{0}=x_{0}^{l} .
$$

Let $\mathcal{J}_{\mathcal{I}}(\mathcal{O}(l))\left(U_{1}\right)$ have the following basis as a left $K[s]$-module:

$$
C:(1,0,0)_{1}=d s \otimes x_{1}^{l},(0,1,0)_{1}=f \otimes x_{1}^{l},(0,0,1)_{1}=x_{1}^{l} .
$$

Theorem 5.5. The structure matrix $[L]_{B}^{C}$ is as follows:

$$
[L]_{B}^{C}=\left(\begin{array}{ccc}
-t^{l-2} & 0 & -l t^{l-1} \\
0 & t^{l} & -l t^{i+l-1} \\
0 & 0 & t^{l}
\end{array}\right) .
$$

Proof. The proof is a straightforward calculation.

We see that the structure matrix $[L]_{B}^{C}$ depends on the integers $i, l$. Maybe one will get examples with more than two different structures of an $\mathcal{O}_{\mathbf{P}}$-module on the locally free sheaf $\mathcal{O}(l-2) \oplus \mathcal{O}(l) \oplus \mathcal{O}(l)$.

Example 5.6. Vector bundles on real and complex manifolds.

Note: If $K$ is the field of complex numbers and $X$ is a smooth projective scheme of finite type over $K$, let $X(\mathbf{R})$ denote the underlying real smooth manifold of $X$. Let $\operatorname{KO}(X(\mathbf{R}))$ be the Grothendieck group of the category of real smooth finite rank vector bundles on $X(\mathbf{R})$. There is a canonical map

$$
\gamma: \mathrm{K}_{0}(X) \rightarrow \mathrm{KO}(X(\mathbf{R}))
$$

sending the class of a locally free finite rank $\mathcal{O}_{X}$-module $\mathcal{E}$ to the class of its underlying finite rank real vector bundle $\mathcal{E}(\mathbf{R})$. In many cases the exact sequence

$$
0 \rightarrow \mathcal{I} \otimes \mathcal{E} \rightarrow \mathcal{J}_{\mathcal{I}}^{1}(\mathcal{E}) \rightarrow \mathcal{E} \rightarrow 0
$$

is split as a sequence of real smooth vector bundles on $X(\mathbf{R})$ since $X(\mathbf{R})$ is a real compact manifold and all short exact sequences of vector bundles split. It follows that there is an isomorphism

$$
\mathcal{J}_{\mathcal{I}}^{1}(\mathcal{E})(\mathbf{R})^{l e f t} \cong \mathcal{J}_{\mathcal{I}}^{1}(\mathcal{E})(\mathbf{R})^{r i g h t}
$$

of real smooth vector bundles. Let

$$
c_{\mathcal{I}}(\mathcal{E})=\left[\mathcal{J}_{\mathcal{I}}^{1}(\mathcal{E})^{l e f t}\right]-\left[\mathcal{J}_{\mathcal{I}}^{1}(\mathcal{E})^{r i g h t}\right] \in \mathrm{K}_{0}(X) .
$$

It follows that there is an equality

$$
\gamma\left(c_{\mathcal{I}}(\mathcal{E})\right)=0
$$

in $\mathrm{KO}(X(\mathbf{R}))$. 
Example 5.7. The classical Atiyah sequence.

Let $\mathcal{E}=\mathcal{O}\left(d_{1}\right) \oplus \cdots \oplus \mathcal{O}\left(d_{e}\right)$ be a locally free sheaf on $\mathbf{P}^{1}$ of rank $e$ over any field $K$. Define

$$
\operatorname{deg}(\mathcal{E})=d_{1}+\cdots+d_{e}
$$

Lemma 5.8. There is an isomorphism

$$
\phi: \mathrm{K}_{0}\left(\mathbf{P}^{1}\right) \cong \mathbf{Z} \oplus \mathbf{Z}
$$

defined by

$$
\phi([\mathcal{E}])=(\operatorname{deg}(\mathcal{E}), r k(\mathcal{E})) .
$$

Proof. By the projective bundle formula there is an isomorphism

$$
\mathrm{K}_{0}\left(\mathbf{P}^{1}\right) \cong \mathbf{Z}[h] /(1-h)^{2}
$$

of rings. We get

$$
\phi\left([\mathcal{O}(-1)]^{d}\right)=h^{d}=(1+h-1)^{d}=1+d(h-1)=1-d t,
$$

where $t$ is the class of $1-h$ in $\mathrm{K}_{0}\left(\mathbf{P}^{1}\right)$. We get similarly

$$
\phi\left([\mathcal{O}(1)]^{d}\right)=1-d(h-1)=1+d(1-h)=1+d t .
$$

It follows that

$$
\phi([\mathcal{E}])=1+d_{1} t+\cdots+1+d_{e} t=r k(\mathcal{E})+\operatorname{deg}(\mathcal{E}) t
$$

and the claim of the lemma follows.

Let $\mathcal{J}^{k}(\mathcal{O}(d))$ be the $k$-th sheaf of principal parts of $\mathcal{O}(d)$ on $\mathbf{P}^{1}$ when $1 \leq k \leq d$. By [10] it follows that

$$
\operatorname{deg}\left(\mathcal{J}^{k}(\mathcal{O}(d))^{l e f t}\right)=\operatorname{deg}\left(\mathcal{J}^{k}(\mathcal{O}(d))^{\text {right }}\right) .
$$

Corollary 5.9. There is an equality

$$
c_{\Omega}(\mathcal{O}(d))=0
$$

in $\mathrm{K}_{0}\left(\mathbf{P}^{1}\right)$.

Proof. By Lemma 5.8 it follows that

$$
\begin{gathered}
c_{\Omega}(\mathcal{O}(d))=\left[\mathcal{J}^{1}(\mathcal{O}(d))^{\text {left }}\right]-\left[\mathcal{J}^{1}(\mathcal{O}(d))^{\text {right }}\right] \\
=\left(\operatorname{rk}\left(\mathcal{J}^{1}(\mathcal{O}(d))^{l e f t}\right), \operatorname{deg}\left(\mathcal{J}^{1}(\mathcal{O}(d))^{\text {left }}\right)\right) \\
-\left(\operatorname{rk}\left(\mathcal{J}^{1}(\mathcal{O}(d))^{\text {right }}\right), \operatorname{deg}\left(\mathcal{J}^{1}(\mathcal{O}(d))^{\text {right }}\right)\right)=0 .
\end{gathered}
$$

The corollary follows.

The triviality of the class $c_{\Omega}(\mathcal{O}(d))$ is related to the fact that the first order jet bundle $\mathcal{J}^{1}(\mathcal{O}(d))$ is an extension of two abelianized $\mathcal{O}_{\mathbf{P}^{1} \text {-modules: }} \Omega_{\mathbf{P}^{1}}^{1} \otimes \mathcal{O}(d)$ and $\mathcal{O}(d)$ : the Atiyah sequence

$$
0 \rightarrow \Omega_{\mathbf{P}^{1}}^{1} \otimes \mathcal{O}(d) \rightarrow \mathcal{J}^{1}(\mathcal{O}(d)) \rightarrow \mathcal{O}(d) \rightarrow 0
$$

is an exact sequence of left and right $\mathcal{O}_{\mathbf{P}^{1}}$-modules and there are isomorphisms

$$
\left(\Omega_{\mathbf{P}^{1}}^{1} \otimes \mathcal{O}(d)\right)^{l e f t} \cong\left(\Omega_{\mathbf{P}^{1}}^{1} \otimes \mathcal{O}(d)\right)^{r i g h t}
$$

and

$$
\mathcal{O}(d)^{l e f t} \cong \mathcal{O}(d)^{r i g h t}
$$


of $\mathcal{O}_{\mathbf{P}^{1} \text {-modules. Hence }}$

$$
\begin{gathered}
c_{\Omega}(\mathcal{O}(d))=\left[\mathcal{J}^{1}(\mathcal{O}(d))^{l e f t}\right]-\left[\mathcal{J}^{1}(\mathcal{O}(d))^{\text {right }}\right] \\
=\left[\left(\Omega_{\mathbf{P}^{1}}^{1} \otimes \mathcal{O}(d)\right)^{l e f t}\right]+\left[\mathcal{O}(d)^{l e f t}\right]-\left[\left(\Omega_{\mathbf{P}^{1}}^{1} \otimes \mathcal{O}(d)\right)^{\text {right }}\right]-\left[\mathcal{O}(d)^{r i g h t}\right]=0 .
\end{gathered}
$$

This argument holds in the following general case.

Theorem 5.10. There is an equality

$$
c_{\Omega}(\mathcal{E})=0
$$

in $\mathrm{K}_{0}(X)$, where $X / S$ is differentially smooth and $\mathcal{E}$ is locally free of finite rank.

Proof. The Atiyah sequence is exact as left and right $\mathcal{O}_{X}$-modules and there are isomorphisms

$$
\left(\Omega_{X}^{1} \otimes \mathcal{E}\right)^{l e f t} \cong\left(\Omega_{X}^{1} \otimes \mathcal{E}\right)^{r i g h t}
$$

of $\mathcal{O}_{X}$-modules.

This example motivates the construction given in this paper: The left and right $\mathcal{O}$-module $\mathcal{I}$ must be nonabelianized for the class $c_{\mathcal{I}}(\mathcal{E})$ to be nontrivial in $\mathrm{K}_{0}(X)$. The class $c_{\mathcal{I}}(\mathcal{E})$ lies in an Ext-group which is computable; hence it should be easy to check if there is an isomorphism

$$
\mathcal{J}_{\mathcal{I}}^{1}(\mathcal{E})^{l e f t} \cong \mathcal{J}_{\mathcal{I}}^{1}(\mathcal{E})^{\text {right }}
$$

of $\mathcal{O}_{X}$-modules.

There is ongoing work where the characteristic class $c_{\mathcal{I}}(\mathcal{E})$ is studied (see [9]).

\section{REFERENCES}

[1] M. André, Homologie des algèbres commutatives, Grundlehren Math. Wiss. no. 206, SpringerVerlag (1974). MR0352220(50:4707)

[2] M. Atiyah, Complex analytic connections in fibre bundles, Trans. Amer. Math. Soc. no. 85 (1957). MR0086359(19:172c)

[3] A. Grothendieck, EGA IV. Étude locale de schémas et des morphismes de schémas, I, Publ. Math. IHES no. 20 (1964). MR0173675(30:3885)

[4] L. Illusie, Complexe cotangent et deformations I, Lecture Notes in Math. Vol. 239, SpringerVerlag (1971). MR0491680(58:10886a)

[5] L. Illusie, Complexe cotangent et deformations II, Lecture Notes in Math. Vol. 283, SpringerVerlag (1972). MR0491681 (58:10886b)

[6] M. Karoubi, Homologie cyclique et $K$-théorie, Astérisque no. 149 (1987). MR913964 (89c:18019)

[7] H. Maakestad, A note on the principal parts on projective space and linear representations, Proc. Amer. Math. Soc., vol. 133, no. 2 (2005). MR2093054 (2005h:14104)

[8] H. Maakestad, Chern classes and Lie-Rinehart algebras, Indagationes Math. no. 18 (2007). MR2424316(2009b:16022)

[9] H. Maakestad, Chern classes and Exan functors, in progress (2009).

[10] H. Maakestad, Principal parts on the projective line over arbitrary rings, Manuscripta Math., vol. 126, no. 4 (2008). MR2425435(2009k:14034)

[11] H. Maakestad, On parameter spaces of right $\mathcal{O}_{X}$-structures, in progress $(2010)$

[12] H. Maakestad, On the structure of jet bundles on projective space, in progress (2011).

Institut Fourier, 100 rue des maths, BP 74, 38402 St. Martin D'Hères cedex, France

E-mail address: h_maakestad@hotmail.com 